\title{
A Quadratic Sieve on the $n$-Dimensional Cube
}

\author{
René Peralta* \\ Electrical Engineering and Computer Science Department \\ University of Wisconsin - Milwaukee
}

\begin{abstract}
Let $N$ be a large odd integer. We show how to produce a long sequence $\left\{\left(X_{2}, Y_{2}\right)\right\}_{i=1}^{2^{n}}$ of integers modulo $N^{r}$ which satisfy $X_{i}^{2} \equiv$ $Y_{2}$ modulo $N$, where $X_{2}>N^{1 / 2}$ and $\left|Y_{2}\right|<c N^{1 / 2}$. Our sequence corresponds to a Hamiltonian path on the n-dimensional hypercube $C_{n}$, where $n$ is $\Theta(\log N / \log \log N)$. One application of these techniques is that, at each vertex of the hypercube, it is possible to search for equations of the form $U^{2} \equiv V$ modulo $N$ with $V$ a product of small primes. The search is as in the quadratic sieve algorithm and therefore very fast. This yields a faster way of changing polynomials in the Multiple Polynomial Quadratic Sieve algorithm, since moving along the hypercube turns out to be very cheap.
\end{abstract}

\section{Introduction}

Given a large odd integer $N$, there is no known way of efficiently generating random congruences of the form $\mathrm{X}^{2} \equiv Y$ modulo $N$ with $Y$ substantially smaller than $N^{1 / 2}$. One reason for wanting to generate such congruences is that they can be used to factor $N$. The Continued Fraction Algorithm [2] factors $N$ by generating many such congruences, choosing the ones for which $Y$ factors over a small prime factor base $F B$, and then solving a linear system of equations in order to create one congruence $X^{2} \equiv Z^{2}$ modulo $N$ which, if $X \neq \pm Z$. vields a proper factor $p=G C D(X+Z, N)$ of $N$. An important bottleneck in the Continued Fraction Algorithm is the cost of testing whether $Y$ factors over $F B$. This is done by trial division for each prime in the factor base. The Quadratic Sieve Algorithm [6] considers a sequence $\left\{\left(Y_{i}, Y_{i}\right)\right\}_{i=1}^{M}$ of $M$ pairs where $X_{i}=\lfloor\sqrt{N}\rfloor+i$ and $Y_{i}=X_{i}^{2}-N$. Since $Y_{i}$ is given by an integer quadratic polynomial, it is easy to predict, which $Y_{i}^{\prime}$ 's will be divisible by a given prime $p$. The values of $i$ which generate $Y_{i} \equiv 0 \bmod p$ lie on two arithmetic progressions $\alpha \pm k p$ and $\beta \pm k p(k=0,1, \ldots)$. The cost of avoiding trial division is that the $Y_{i}$ 's are of order $O\left(M N^{1 / 2}\right)$ and therefore they are less likely to factor over $F B$ than the $Y$ 's generated by the Continued Fraction Algorithm. However, avoiding trial division more than compensates for the increased size of the $Y_{i}$ 's. A variation on the Quadratic Sieve Algorithm is the Multiple Polynomial Quadratic Sieve [8] (MPQS), which uses several polynomials as a way to fight the increase in the size of the $Y_{i}$ 's. The latter is currently the algorithm of choice for factoring integers which are about one hundred digits long.

\footnotetext{
* Supported by National Science Foundation grant No. C(CR-8909657 
We show how to produce a long sequence $\left\{\left(X_{i}, Y_{i}\right)\right\}_{i=1}^{2^{n}}$ of integers modulo $N$ which satisfy $X_{i}^{2} \equiv Y_{i}$ modulo $N$, where $X_{i}>N^{1 / 2}$ and $\left|Y_{i}\right|<c N^{1 / 2}$. Our sequence corresponds to a Hamiltonian path on the $n$-dimensional hypercube $C_{n}$, where $n$ is $\Theta(\log N / \log \log N)$. One application of these techniques is that, at each vertex of the hypercube, it is possible to search for equations of the form $U^{2} \equiv V$ modulo $N$ with $V$ smooth. The search is as in the quadratic sieve algorithm and therefore very fast. This yields a factoring algorithm which is faster than the Multiple Polynomial Quadratic Sieve algorithm, since moving along the hypercube turns out to be very cheap. The asymptotics of the new algorithm are as in MPQS. Therefore it is not asymptotically as fast as the recently discovered Number Field Sieve algorithm [3, 1].

\section{Generating "small" quadratic congruences}

Let $N$ be a large odd integer. We will use the symbol " $\equiv$ " to denote modular congruence, and we will restrict the use of " $=$ " to equality. Let $s, t$ be such that

$-t=\prod_{j=1}^{n} p_{j}$, where the $p_{j}$ 's are distinct primes ( $n$ will be chosen later).

- The prime 2 may be among the $p_{j}$ 's if and only if $N \equiv 1$ modulo 4 .

$-N$ is a quadratic residue modulo each $p_{j}$.

- $s$ satisfies $s^{2} \equiv N$ modulo $t^{2}$ and $|s|<t^{2}$.

Lemma 1. Let $c=t / N^{1 / 4}$. Let $x \equiv s / t \operatorname{modulo} N$ and $y \boxminus x^{2} \operatorname{modulo} N$ where $y$ is the member of the residue class of $x^{2}$ with smallest absolute value. Then $|y|<c_{1} N^{1 / 2}$ where $c_{1}=\operatorname{Max}\left\{c^{2}-\frac{1}{c^{2}}, \frac{1}{c^{2}}\right\}$.

Proof. Since $s^{2} \equiv N$ modulo $t^{2}$, we have $s^{2}=k t^{2}+N$ for some (possibly negative) integer $k$. Then $y \equiv \frac{s^{2}}{t^{2}} \equiv \frac{s^{2}-N}{t^{2}}=\frac{k t^{2}+N-N}{t^{2}}=k$, where congruence is modulo $N$. Thus we may choose $y=k$. Since $s^{2}<t^{4}$ and $t=c N^{1 / 4}$, we have

$$
|y|=|k|=\left|\frac{s^{2}-N}{t^{2}}\right| \leq \operatorname{Max}\left\{t^{2}-\frac{N}{t^{2}}, \frac{N}{t^{2}}\right\}=N^{1 / 2} \operatorname{Max}\left\{c^{2}-\frac{1}{c^{2}}, \frac{1}{c^{2}}\right\} . \square
$$

Thus, if $c \in\left(1, \sqrt{\frac{1+\sqrt{5}}{2}}\right)$, then $|y|<N^{1 / 2}$ (the golden mean strikes again!). Also note that $\operatorname{Max}\left\{c^{2}-\frac{1}{c^{2}}, \frac{1}{c^{2}}\right\}$ is minimized at $c=2^{1 / 4}$. For $c<2^{1 / 4}$, the bound is $\frac{1}{c^{2}}$. For $c>2^{1 / 4}$, the bound is $c^{2}-\frac{1}{c^{2}}$.

By construction, there are $2^{n}$ square roots of $N$ modulo $t^{2}$. Given the $p_{j}$ 's, it is a simple matter to compute one such root $s_{1}$. By the Chinese Remainder Theorem, we may think of $s_{1}$ as an $n$-tuple $\left(\alpha_{1}, \ldots, \alpha_{n}\right)$, where $\alpha_{j}^{2} \equiv N$ modulo $p_{j}^{2}$. Then the complete set of square roots of $N$ modulo $t^{2}$ is given by $\left( \pm \alpha_{1}, \ldots, \pm \alpha_{n}\right)$ for all choices of signs \pm . Any member of this set can be easily calculated as a $\operatorname{sum} \sum_{j=1}^{n} \delta_{j} \alpha_{j} b_{j}$ modulo $t^{2}$ where

$-\delta_{j}$ is the sign at the $j$-th coordinate.

$-b_{j}$ is the unique element of $Z_{t^{2}}$ which is 1 modulo $p_{j}^{2}$ and 0 modulo $p_{i}^{2}$ for $j \neq i$. 
Note that there are two possible values for each $\alpha_{j}$. We will choose $\alpha_{j}$ such that $b_{j} \alpha_{j}$ modulo $t^{2}$ is less than $\frac{t^{2}}{2}$.

The maximum size of $n$ can be estimated from the familiar relation $\sum_{p \leq x} \ln p$ $\sim x$, where the sum is over all primes $p$ less than or equal to $x$. Since $N$ is typically a quadratic residue modulo half of the first $2 n$ primes we can estimate. the maximum $n$ from $2 n \sim \pi(x)$ where $x$ satisfies $\prod_{p \leq x} p=N^{1 / 2}$ (in this way, the product of the approximately $n$ primes for which $N$ is a quadratic residue is approximately $\left.N^{1 / 4}\right)$. This implies $\sum_{p \leq x} \ln p \sim \frac{1}{2} \ln N$, and so $x \sim \frac{1}{2} \ln N$. Thus $n \sim \frac{1}{2} \pi(x) \sim \frac{1}{2} \pi\left(\frac{1}{2} \ln N\right) \sim \frac{1}{4} \frac{\ln N}{\ln \ln N-\ln 2}=\Theta(\log N / \log \log N)$.

\section{Example: the RSA modulus}

The 129-digit R.SA modulus

$$
N_{R S A}=114381625757888867669235779976146612010218296721242362562561
$$

842935706935245733897830597123563958705058989075147599290026879543541 is a quadratic residue modulo the 20 primes

$$
\{2,5,17,19.29,37,41,43,47,59,79,97,101,103,107.113,131.151,157,163\} .
$$

Letting $t$ be the product of all these primes except 79 , we get $t \sim 1.01 \mathrm{~V}_{\mathrm{RSA}}^{1 / 4}$ Thus, for this case we get $n=19$.

\section{Traversing the hypercube}

The set of square roots of $N$ modulo $t^{2}$ can be thought of as the n-dimensional hypercube, where we connect two roots if and only if they differ at exactly one sign. A Hamiltonian path on the hypercube is defined by a starting point $s_{1}$ and the sequence $\left\{k_{i}\right\}_{i=1}^{2^{n}-1}$ of coordinate changes, e.g. $k_{130}=8$ means the 130 th move on the Hamiltonian path is a change of sign at coordinate 8 . Let $\mu_{i}=+1$ if move $i$ switches a $-\operatorname{sign}$ for $a+\operatorname{sign}$ and $\mu_{i}=-1$ if move $i$ switches a + sign for a - sign. Let $\gamma_{j} \equiv \alpha_{j} b_{j}$ modulo $t^{2}$, where $\alpha_{j}, b_{j}$ are as defined in the previous section. Note that, by our choice of $\alpha_{j}$, we have $0<\gamma_{j}<\frac{t^{2}}{2}$ for all $j$. Then we may define the $i$-th square root of $N$ modulo $t^{2}$ by

$$
s_{i+1}=s_{i}+2 \mu_{i} \gamma_{k_{i}}-\omega_{i} t^{2}
$$

where $\omega_{i}$ is a correction factor to make $s_{i} \in\left(0, t^{2}\right)$. Note that $\omega_{i}$ is always $-1,0$, or +1 . The sequence of $\omega_{i}$ 's can be easily computed from the few most significant bits of the $\gamma_{j}$ 's, and if simply allowed to be 0 , the values of $s_{i}$ will remain in a small interval (as shown by our next example).

An $n$-dimensional cube $C_{n}$ is composed of two $(n-1)$-dimensional cubes $C_{n-1}^{(1)}, C_{n-1}^{(2)}$ whose vertices are connected in a $1-1$ fashion. Thus, a simple way to traverse the $n$-cube is 
- traverse $C_{n-1}^{(1)}$

- move to $C_{n-1}^{(2)}$

- traverse $C_{n-1}^{(2)}$.

The recursive procedure works because on moving to $C_{n-1}^{(2)}$ the algorithm finds itself at a node which is, up to isomorphism, the same starting node as in $C_{n-1}^{(1)}$. From now on we will assume the Hamiltonian path on the n-cube is generated by this procedure.

Example: Traversing the 3-cube

A traversal of the 3-cube yields

\begin{tabular}{|c|c|c|c|c|c|c|c|}
\hline $\mathrm{i}$ & 1 & 2 & 3 & 4 & 5 & 6 & 7 \\
\hline$k_{i}$ & 1 & 2 & 1 & 3 & 1 & 2 & 1 \\
\hline$\mu_{i}$ & -1 & -1 & +1 & -1 & -1 & +1 & +1 \\
\hline
\end{tabular}

Csing $\omega_{i}=0$ for all $i$, this table gives the following values for the $s_{i}$ 's.

$$
\begin{aligned}
s_{2} & =s_{1}-2 \gamma_{1} \\
s_{3} & =s_{2}-2 \gamma_{2} \\
& =s_{1}-2\left(\gamma_{1}+\gamma_{2}\right) \\
s_{4} & =s_{3}+2 \gamma_{1} \\
& =s_{1}-2 \gamma_{2} \\
s_{5} & =s_{4}-2 \gamma_{3} \\
& =s_{1}-2\left(\gamma_{2}+\gamma_{3}\right) \\
s_{6} & =s_{5}-2 \gamma_{1} \\
& =s_{1}-2\left(\gamma_{1}+\gamma_{2}+\gamma_{3}\right) \\
s_{7} & =s_{6}+2 \gamma_{2} \\
& =s_{1}-2\left(\gamma_{1}+\gamma_{3}\right) \\
s_{8} & =s_{7}+2 \gamma_{1} \\
& =s_{1}-2 \gamma_{3}
\end{aligned}
$$

The value of $s_{6}$ could be larger in absolute value than $2 t^{2}$, but no larger than $3 t^{2}$. This illustrates the point that if the $\omega_{i}$ 's are not used then $s_{i}$ still remains in the interval $\left(-n t^{2}, n t^{2}\right){ }^{2}$ Also note that the sequence of $k_{i}$ 's can be generated

${ }^{2}$ Different Hamiltonian paths on the hypercube might yield different bounds. 
in linear time (to generate the sequence for $C_{n}$ simply put $n$ between two copies of the sequence for $(n-1)$.

Thus the integer recurrence

$$
s_{i+1}=s_{i}+2 \mu_{i} \gamma_{k_{1}}-\omega_{i} t^{2}
$$

together with

$$
x_{i} \equiv\left(s_{i} / t\right) \text { modulo } N ; y_{i} \equiv\left(x_{i}\right)^{2} \text { modulo } N
$$

yield

$$
\left|y_{i}\right|<\operatorname{Max}\left\{c^{2}-\frac{1}{c^{2}}, \frac{1}{c^{2}}\right\} N^{1 / 2}
$$

if $y_{i}$ is the member of the residue class of $x_{i}^{2}$ with smallest absolute value. We will now produce an integer recurrence for the $y_{i}$ 's.

Note that, modulo $N$.

$$
\begin{aligned}
y_{i+1} & \equiv\left(s_{i+1} / t\right)^{2} \\
& \equiv\left(\frac{s_{i}+2 \mu_{i} \gamma_{k_{i}}-\omega_{i} t^{2}}{t}\right)^{2} \\
& \equiv\left[\left(\frac{s_{i}}{t}\right)^{2} \operatorname{modulo} V_{j}+\frac{\left(2 \mu_{i} \gamma_{k_{i}}-\omega_{i} t^{2}\right)^{2}}{t^{2}}+\frac{2 s_{i}\left(2 \mu_{i} \gamma_{k_{2}}-\omega_{i} t^{2}\right)}{t^{2}}\right. \\
& \equiv y_{i}+\frac{\left(2 \mu_{i} \gamma_{k_{i}}-\omega_{i} t^{2}\right)^{2}}{t^{2}}+\frac{2 s_{i}\left(2 \mu_{i} \gamma_{k_{i}}-\omega_{i} t^{2}\right)}{t^{2}}
\end{aligned}
$$

Since $s_{i}^{2} \equiv N$ modulo $t^{2}$ and $s_{i+1}^{2} \equiv V$ modulo $t^{2}$, we have $\left(2 \mu_{i} \gamma_{k}\right.$, $\left.\omega_{i} t^{2}\right)^{2}+2 s_{i}\left(2 \mu_{i} \gamma_{k_{2}}-\omega_{i} t^{2}\right)$ is congruent to 0 modulo $t^{2}$. Thus

$$
\frac{\left(2 \mu_{i} \gamma_{k_{i}}-\omega_{i} t^{2}\right)^{2}}{t^{2}}+\frac{2 s_{i}\left(2 \mu_{i} \gamma_{k_{1}}-\omega_{i} t^{2}\right)}{t^{2}}
$$

is an integer which is easily seen to be of order $N^{1 / 2}$. This means the integer recurrence

$$
y_{i+1}=y_{i}+\frac{\left(2 \mu_{i} \gamma_{k_{1}}-\omega_{i} t^{2}\right)^{2}}{t^{2}}+\frac{2 s_{i}\left(2 \mu_{i} \gamma_{k_{i}}-\omega_{i} t^{2}\right)}{t^{2}}
$$

holds. By lemma 1, $y_{1}$ can be chosen so that $\left|y_{1}\right|<c_{1} N^{1 / 2}$ where $c_{1}=\operatorname{Max}\left\{c^{2}-\right.$ $\left.\frac{1}{c^{2}}, \frac{1}{c^{2}}\right\}$. Again by lemma 1, the integer recurrence generates $y_{i}$ 's whose absolute value is less than $\operatorname{Max}\left\{c^{2}-\frac{1}{c^{2}}, \frac{1}{c^{2}}\right\} N^{1 / 2}$.

Now let us traverse the hypercube "modulo $p$ ", where $p$ is a small prime. This simply means generating the sequence of $y_{i}$ 's modulo $p$. Assume $p$ is not a factor of $t$. We may write.

$$
\begin{gathered}
y_{i+1}=y_{i}+\Psi_{i}+s_{i} \Upsilon_{i} \\
s_{i+1}=s_{i}+\Delta_{i}
\end{gathered}
$$


where

$$
\Psi_{i}=\frac{\left(2 \mu_{i} \gamma_{k_{i}}-\omega_{i} t^{2}\right)^{2}}{t^{2}} \quad ; \quad Y_{i}=\frac{2\left(2 \mu_{i} \gamma k_{i}-\omega_{i} t^{2}\right)}{t^{2}} \quad ; \quad \Delta_{i}=\left(2 \mu_{i} \gamma_{k_{i}}-\omega_{i} t^{2}\right) .
$$

Note that $\Psi_{i}, \Upsilon_{i}$ and $\Delta_{i}$ can take on at most $6 n$ values ( $\mu_{i}$ can take on two values, $\omega_{i}$ can take on three values, and $\gamma_{k}$ can take on $n$ values). Thus, $\Psi_{i}, \Upsilon_{i}$ and $\Delta_{i}$ can be read from precomputed tables, of size $6 n$ and indexed by $\mu_{i}, \omega_{i}, k_{i}$. Thus, computing $\left(y_{i+1}, s_{i+1}\right)$ modulo $p$ from $\left(y_{i}, s_{i}\right)$ modulo $p$ involves one multiplication and three additions modulo $p$. The cost of computing $s_{i+1} \bmod p$ from $s_{2} \bmod p$ is one addition modulo $p$. This fact will be used in section 4 .

Note that precomputation is not possible if $p$ divides $t$, since then $\bar{\psi}_{i}$ and $\Upsilon_{i}$ may not be defined modulo $p$. More specifically, $\Psi_{i}, \Upsilon_{i}$ are not defined modulo $p_{k_{i}}$.

Also note that, if $N$ is not a quadratic residue modulo $p$, then $p$ does not divide $y_{i}$. This can be shown as follows: Suppose $N$ is not a quadratic residue modulo $p$. Then $p$ does not divide $t^{2}$ and therefore $p$ divides $y_{i}=\frac{s^{2}-N}{t^{2}}$ if and only if $p$ divides $s_{i}^{2}-N$. But if this was the case then $s_{i}^{2} \equiv N$ modulo $p$, which would contradict the assumption that $N$ is not a quadratic residue modulo $p$.

\section{A factoring algorithm}

The algorithm consists of visiting A vertices of the hypercube and, at each vertex $s$, finding the values of $\lambda$ for which

$$
z_{\lambda}=(s / t+\lambda t)^{2} \bmod \lambda \quad(\lambda \in-M . M)
$$

is $B$-smooth. The optimal values of $A . B$, and $M$ will follow from the analysis of the algorithm. We actually do not compute the $z_{\lambda}$, but rather find the values of $\lambda$ for which $z_{\lambda}$ is $B-$ smooth. So that the $z_{\lambda}$ are "small". we will choose $t \sim \frac{N^{1 / 4}}{\sqrt{M}}$.

Notice that

$$
\tilde{z}_{\lambda} \equiv(s / t)^{2}+\lambda^{2} t^{2}+2 s \lambda \bmod N=\frac{s^{2}-N}{t^{2}}+\lambda^{2} t^{2}+2 s \lambda .
$$

Thus we set

$$
\frac{s^{2}-N}{t^{2}}+\lambda^{2} t^{2}+2 s \lambda \equiv 0 \bmod p
$$

This yields

$$
\lambda \equiv(-s \pm \sqrt{N}) t^{-2} \bmod p
$$

where $\sqrt{N}$ is a modular square root. Therefore $z_{\lambda}$ is divisible by $p$ for all $\lambda=$ $k p+D_{s}$ and all $\lambda=k p+E_{s}$, where

$-k$ is an integer:

$-D_{s}=(-s+\sqrt{N}) t^{-2} \bmod p ;$

$-E_{s}=(-s-\sqrt{N}) t^{-2} \bmod p=D_{s}-2 \sqrt{N} p^{-2} \bmod p$. 
Thus, the "good" $\lambda$ modulo $p$ are in arithmetic progressions. Therefore standard sieving techniques can be used to find those $\lambda$ for which $z_{\lambda}$ is $B$-smooth.

We may precompute $\sqrt{N} \bmod p, t^{-2} \bmod p$, and $-2 \sqrt{N} t^{-2} \bmod p$. Therefore computing $D_{s}$ and $E_{s}$ involves

- one addition to compute $s$ mod $p$ (see section 3 );

- one addition and one multiplication to compute $D_{s}$;

- one addition to compute $E_{s}$.

Thus the total cost of moving from one vertex of the hypercube to another is, essentially, three additions and one multiplication modulo $p$ for each prime in the factor base. This is much cheaper than the cost of changing polynomials in the Multiple Polynomial Quadratic Sieve.

We now show that the $z_{\lambda}$ 's are about $M N^{1 / 2}$ in absolute value. Recall that $t \sim \frac{N^{1 / 4}}{\sqrt{M}}$ and consider

$$
\tilde{\sim}_{\lambda} \equiv(s / t+\lambda t)^{2} \equiv(s / t)^{2}+\lambda^{2} t^{2}+2 s \lambda \bmod N
$$

As in the proof of lemma 1 we have $(s / t)^{2} \bmod N=k$ where $s^{2}=k t^{2}+N$. Since $s^{2}<t^{4}<N$. we have that $k$ is negative. By lemma $1,\left|(s / t)^{2} \bmod V\right|=|k| \leq$ $M N^{1 / 2}$. Assuming, for simplicity, that $t<\frac{N^{1 / 4}}{\sqrt{M}}$, we have $\lambda^{2} t^{2} \leq M^{2} \frac{N^{1 / 2}}{M}=$ $M N^{1 / 2}$. Since $s<i^{2}$. We have $|2 s \lambda|<2 \frac{N^{1 / 2}}{M} M=2 N^{1 / 2}<<M V^{1 / 2}$. Thus $z_{\lambda}$ is, essentially, the difference of two numbers in the range $0 \ldots M N^{1 / 2}$. We conclude that our algorithm is faster than the Multiple Polynomial Quadratic Sieve because

- vertices in the hypercube correspond to polynomials in MPQS.

- for each vertex, the cost of sieving $2 M$ locations is the same in our algorithm as in MPQS.

- the size of the quadratic residues considered is, as in MPQS, about $M N^{1 / 2}$.

- changing polynomials is much more expensive than changing vertices of the hypercube. Therefore the optimal value for the size of the hypercube path is bigger than the optimal number of polynomials in MPQS. This means that $M$ will be smaller in our algorithm and therefore $M N^{1 / 2}$ will be smaller. Thus, our algorithn will generate smaller quadratic residues than MPQS.

In practice there are many speedups to be included in an implementation of this factoring algorithm. All the enhancements described in [5] can be used with this algorithm. A rough estimate of how much faster this algorithm is than MPQS can be obtained as follows:

Let $T$ be the ruming time of the algorithm. in terms of arithmetic operations on single-precision numbers. Let $V$ be the cost of moving from one vertex to another. Let $S$ be the cost of sieving at each vertex. Suppose we sieve modulo all primes less than $B$ for which $N$ is a quadratic residue. There are about $\frac{\pi(B)}{2}$ such primes. It takes four operations per prime to make a move on the hypercube. Therefore we can estimate $V$ by $2 \pi(B)$. We can estimate $S$ by $1 / 2 \sum_{p<B} 4 M / p$ since for each prime $p$ in the factor base about $4 M / p$ locations of an accumulator 
array need be updated. We can estimate this surn by $6 M$. Thus our estimate for $T$ is $A(S+V)=2 A \pi(B)+6 A M$.

Let, $F(y, x)$ be the probability that a random number in $Z_{y}$ factors over primes smaller than $x$. The number of quadratic residues considered by our algorithm is $2 A M$, and each can be thought of as a random number in $Z_{\left\lfloor M^{1 / 2}\right\rfloor}$. Thus about $2 A M F\left(M N^{1 / 2}, B\right)$ of the quadratic residues will be $B$-smooth. Since we need about $\pi(B) / 2$ smooth quadratic residues, we set

$$
2 A M F\left(M N^{1 / 2}, B\right)=\pi(B) / 2 \text {. }
$$

Approximating $\pi(B)$ by $B / \ln B$ and $F(y, x)$ by $(\ln x / \ln y)^{\ln y / \ln x}$ (see [4]), our problem is to minimize

$$
2 A B / \ln B+6 A M
$$

subject to

$$
A=\frac{B}{4 M \ln B}\left(\frac{\ln \left(M N^{1 / 2}\right)}{\ln B}\right)^{\frac{\ln \left(M N^{1 / 2}\right)}{\ln B}}
$$

The solution to this optimization problem can be approximated numerically. For $N \sim 10^{100}$, optimal values are

$$
A=1.2 \cdot 10^{5} ; B=1.4 \cdot 10^{8} ; M=1.3 \cdot 10^{7} ; T=1.1 \cdot 10^{13}
$$

Assuming the cost of changing pnlynomials in MPQS is $50 \pi(B),{ }^{3}$ the numbers for MPQS are

$$
A=5.8 \cdot 10^{3}: B=1.9 \cdot 10^{\times}: M=4.5 \cdot 10^{8}: T=1.9 \cdot 10^{13}
$$

Thus it appears that our techniques significantly improve on the running time of MPQS.

\subsection{Remarks}

1. The running-time predictions given above are very crude estimates. The true test of the running time of this algorithm will be its implementation.

2. In practice, the $\omega_{i}$ 's defined in section 2 can be set to zero without a significant cost in the running time of the algorithm. Doing so has the advantage of diminishing the memory requirements of the algorithm.

3 . In practice, the factors of thould not be small primes. This is because the numbers being sieved have a chance of $1 / p$ of being divisible by $p$ when $p$ divides $t$ (as opposed to $2 / p$ when $p$ is an odd prime in the factor base which does not divide $t$ ). The resulting loss of smoothness is significant for small $p$. Because of this, the $t$ we use in practice may not have as many factors as the optimization of parameters requires.

\footnotetext{
3 This is 25 times as expensive as in our algorithm. Changing polynomials in MPQS involves arithmetic with large numbers. Hence the cost will depend on the particular implementation of large number arithmetic. The number 25 was arrived at using "ln $++"$, a c ++ package developed at UWM.
} 
4. Pomerance, Smith. and Tuler [7], and Montgomery (reported in [7]) propose ways of speeding up MPQS which are similar to the one proposed here. Their methods can be combined with the techniques being proposed bere. It appears that doing so may further improve the running time of the algorithm.

5. The number of vertices of the hypermbe to be visited by the factoring algorithm should be at most $2^{n-1}$, where $n$ is the number of factors of $i$. Otherwise duplication of polynomials orcurs, since $(s / t+\lambda t)^{2}$ and $\left(\left(t^{2}-s\right) / t+\lambda t\right)^{2}$ are essentially equivalent.

\section{Acknowledgments}

I am indebted to anonymons referees for numerous suggestions, inchinding remarks 3 and 4 above.

\section{References}

1. L.M. Adleman. Factoring numbers using singular integers. In Procedings of the 2.3th Annual d C S Symposium on the Theory of romputing pages 64-71, 1991.

2. D.H. Jehmer and R.E. Powers. On factoring large numbers. Bull. Amer. Math. Soc.. 37:770-776, 1931.

3. A.K. Lenstra. H. W. lenstra, M.S. Manasse and J.M. Pollard. The number field sieve. In Procedings of the Din Annual ACM Symposiam on the Theory of Computirg, pages $564-572.1990$.

4. D. Knuth and L. Trabu Pardo. Analysis of a simple factorization algorithm. Theoretical Computer Science, 3:321-348, 1976.

5. A.K. Lenstra and M.S. Manasse. Factoring by electronic mail. In Advances in Cryptology-procedings of ElRORRYPT 89 , volume 434, pages 355-371. SpringerVerlag, 1990

6. C. Pomerance. Analysis and comparison of some integer factoring algorithms. $154: 89-139,1982$

7. C. Pomerance. J.W. Sinith, and R. Tuler. A pipeline architecture for factoring large integers with the quadratic sieve algorithm. SLAW Journal on Computing, $17(2): 387-403,1988$.

8. R. Silverman. The multiple polynomial quadratic sieve. Mathematics of Computation, $48(177): 329-339$. 1987 . 